\title{
A STRONG INFRARED RADIATION FROM MOLECULAR NITROGEN IN THE NIGHT SKY
}

\author{
Joel Stebbins, ${ }^{1}$ A. E. Whitford, ${ }^{2}$ and P. Swings \\ Mount Wilson Observatory and Washburn Observatory \\ Received October 23, 1944
}

\section{ABSTRACT}

A new infrared radiation has been detected in the night sky, which is far more intense than the ordinary persistent aurora giving the green line at $5577 \mathrm{~A}$. Measured with a photocell and filters, the wave length of the new radiation is $10,440 \pm 25 \mathrm{~A}$.

This night-sky radiation is identified with the $(0,0)$ band of the first positive group $B^{3} \Pi \rightarrow A^{3} \Sigma$ of $N_{2}$. The absence of other $N_{2}$ bands suggests that emission of the $(0,0)$ band involves conversion of the energy of dissociation $D\left(N_{2}\right)$ into excitation in a three-body collision:

$$
N+N+N_{2} \rightarrow N_{2}+N_{2}^{\text {exo }} \text {. }
$$

Since $D\left(N_{2}\right)$ is a little larger than the excitation energy of $B^{3} \Pi, v^{\prime}=0$, but smaller than $\mathrm{B}^{3} \Pi, v^{\prime}=1$, only the bands arising from $\mathrm{B}^{3} \Pi, v^{\prime}=0$, would be excited; and of the latter, only $(0,0)$ is observable. This mechanism implies the presence of a large number of nitrogen atoms in the high atmosphere. It can be effective only with the value 7.38v. of $D\left(N_{2}\right)$ advocated by Herzberg and Sponer.

In the year 1940 a program of measures of stars and nebulae in different spectral regions was begun by Stebbins and Whitford. With a photoelectric cell and suitable filters it was possible to isolate six regions ranging from $3530 \mathrm{~A}$ to $10,300 \mathrm{~A}$. The observations were made with the 60 -inch and 100-inch reflectors; and for measures of large objects, like the nebulae, the photometer was equipped with focal diaphragms up to $19 \mathrm{~mm}$ in diameter, giving an angular field of 8.6 on the 60 -inch and 5.1 on the 100 -inch reflector.

TABLE 1

COMPARISON OF STAR AND SKY

\begin{tabular}{c|c|c|c|c|c|c}
\hline \hline Filter & $\begin{array}{c}\text { Ultraviolet } \\
\lambda 3530\end{array}$ & $\begin{array}{c}\text { Violet } \\
\lambda 4220\end{array}$ & $\begin{array}{c}\text { Blue } \\
\lambda 4880\end{array}$ & $\begin{array}{c}\text { Green } \\
\lambda 5700\end{array}$ & $\begin{array}{r}\text { Red } \\
\lambda 7190\end{array}$ & $\begin{array}{r}\text { Infrared } \\
\lambda 10,300 \mathrm{~A}\end{array}$ \\
\hline Deflection, star............... & 8 & 8 & 10 & 8 & 8 & $8 \mathrm{~mm}$ \\
Deflection, sky..... & 8 & 5 & 5 & 8 & 13 & $112 \mathrm{~mm}$ \\
\hline
\end{tabular}

On the very first exposure to a nebula, $M$ 31, through an infrared filter giving an effective wave length of $10,300 \mathrm{~A}$, the galvanometer deflection was unexpectedly large. A check exposure to neighboring blank sky, to determine the background effect, promptly showed that there was a strong infrared radiation in the night sky which would have to be eliminated from any measures of objects of low surface brightness, like the nebulae. This elimination of the sky background is readily accomplished by sliding the photometer on the telescope from object to blank sky and then back to object, this being the regular observing procedure for nebulae and clusters and for faint stars where with even the smallest practical diaphragm the relative sky effect is appreciable.

As a sample of the relative effects between a star and the sky, we have the data in Table 1. A star of type dF7 was selected as giving roughly the same deflection for each

${ }^{1}$ Research Associate of the Mount Wilson Observatory, Carnegie Institution of Washington.

${ }^{2}$ On leave at Massachusetts Institute of Technology. 
spectral region with the cell and filters used. The infrared radiation is obviously much brighter than any other part of the spectrum, including the persistent auroral line at $5577 \mathrm{~A}$, for which we have never found any effect. With allowance for the continuous sky spectrum, the infrared radiation is probably scores of times-perhaps even a hundred times-as strong as the line in the green. At first we thought that the infrared effect was caused by the line at 8600 A discovered by V. M. Slipher, ${ }^{3}$ but it soon became evident that this assumption could not be true. An inspection of the curves for these filters 4 shows that the response at $8600 \mathrm{~A}$ should be greater for the red than for the infrared filter and that, while the increased sky deflection for the red may be caused by the line at $8600 \mathrm{~A}$, the main source must be farther out, probably near 10,000 A, where it would be between the strong water-vapor absorption bands $\rho$ and $\Phi$, roughly at $9500 \mathrm{~A}$ and $11,300 \mathrm{~A}$, respectively. The decreasing sensitivity of the photocell makes improbable anything beyond $11,500 \mathrm{~A}$.

That the new radiation must be atmospheric was shown by its variation with the zenith distance, by its usual decrease through the night, and by its irregular variability from night to night and from season to season. Most of the measures were made with the 60 -inch reflector incidentally to observations of stars and nebulae, for it never seemed worth while to devote the time of a large reflector to work which could be done with any $f: 5$ condensing lens or even by pointing the photometer directly at the sky. But, despite the fragmentary character of our data (only three or four measures per night), a rough impression of the changes in this radiation could be formed. The brightness is greatest immediately after twilight, when, under standard conditions, an exposure to the sky near the pole with a $12-\mathrm{mm}$ focal diaphragm on the 60 -inch gives a galvanometer deflection of 100-150 mm. In general, this intensity decreases through the night until the deflection is, say, 70-100 $\mathrm{mm}$ before dawn.

During the night the telescope is being pointed to all parts of the sky; and the measurements show that the infrared intensity varies with the zenith distance. The intensity probably varies in proportion to $\sec z$; but because of erratic changes in intensity this law could be more easily tested with a smaller telescope. Measures should be made at widely different zenith distances at intervals of time not greater than a minute and should be carried to near the horizon in order to estimate the height of the emitting layer above the earth's surface. All this sounds simple enough; but with the 60 -inch the program on stars and nebulae includes measures in six colors, and the sky background must be eliminated in all six. There are also further complications when the moon is above the horizon. At that time the sky is bluer, as shown by the increased intensity in the ultraviolet, with progressively less change toward the infrared.

One night we had occasion to test the infrared sky during the approach of dawn. With the telescope pointed at the zenith, the galvanometer deflections increased in 20 minutes from $70 \mathrm{~mm}$ to a sharp maximum of $94 \mathrm{~mm}$ and then decreased to $66 \mathrm{~mm}$ by 20 minutes later. The maximum occurred within one minute of the computed time for the sun $18^{\circ}$ below the horizon. This dependence of the infrared upon the incidence of solar radiation in the upper atmosphere should, of course, be investigated near both suirise and sunset.

Renewed interest was awakened on July 20,1944, when the infrared sky was found unexpectedly to be of nearly twice its ordinary brightness and to be fluctuating by 10 or 15 per cent within 10 minutes. Near midnight the deflections were up to about $200 \mathrm{~mm}$, then dropped back to $100 \mathrm{~mm}$ before dawn. We seemed to be witnessing an infrared auroral display, but there was no disturbance of the magnetometer at Mount Wilson that night. By two nights later the deflections were back to normal or below; on a night in

${ }^{3} M . N ., 93,666,1933$. In a private communication Dr. Slipher states that he has detected other radiations near 10,000 A, but he has not determined the wave lengths. Whether or not his spectrograms extend out to $10,450 \mathrm{~A}$ is not clear; possibly they do.

${ }^{4}$ Stebbins and Whitford, Mt.W. Contr., No. 680, p. 4; Ap. J., 98, 23, 1943. 
September, however, the deflections got up to $250 \mathrm{~mm}$ without minute-to-minute fluctuations.

In the course of a discussion of these outbursts in the night sky P. Swings suggested that the radiation was undoubtedly molecular in origin and very likely could be traced to the $(0,0)$ band of nitrogen at about $10,450 \mathrm{~A}$. With this suggestion as a guide, it was proposed to determine the wave length in some way with the photocell and a suitable filter, since a photograph of the sky spectrum at 10,450 A would require an extremely long exposure. For the Schott filter UG6, $2 \mathrm{~mm}$ thick, the following transmissions are given by the maker (reflections neglected):

$$
\begin{array}{rrrr}
8500 \mathrm{~A} \ldots \ldots \ldots & 0.01 & 11,500 \mathrm{~A} \ldots \ldots \ldots & 0.79 \\
9500 \ldots \ldots \ldots & .25 & 13,000 \ldots \ldots \ldots & 0.92 \\
10,500 \ldots \ldots \ldots & 0.59 & &
\end{array}
$$

One piece of this filter should absorb all wave lengths shorter than $8500 \mathrm{~A}$; then, if the sky radiation is confined to a band near $10,000 \mathrm{~A}$, each additional piece would transmit the same fraction of the light incident upon that piece.

Measures of the sky were obtained on several nights, giving the respective transmission ratios, two filters to one and three filters to one:

$$
\begin{aligned}
& (2) /(1)=0.565 \pm 0.007 \text { (p.e.) } \\
& (3) /(1)=0.348 \pm 0.005
\end{aligned}
$$

and, dividing $(b)$ by $(a)$,

$$
(3) /(2)=0.616 \pm 0.012 \text {. }
$$

With only four filters available it was not convenient to measure $(c)$ directly, but from the difference between $(a)$ and $(c)$ we conclude that there must be some radiation in addition to a narrow band. If the extra light is of longer wave length than the assumed

TABLE 2

CALIBRATION OF FILTERS

\begin{tabular}{l|c|c|c}
\hline \hline & $10,336 \mathrm{~A}$ & $10,407 \mathrm{~A}$ & $10,478 \mathrm{~A}$ \\
\hline Observed $(2) /(1) \ldots \ldots \ldots \ldots \ldots \ldots$ & $0.508 \pm 0.024$ & $0.564 \pm 0.010$ & $0.589 \pm 0.020$ \\
Observed $(3) /(1) \ldots \ldots \ldots \ldots \ldots$ & $0.264 \pm 0.000$ & $0.318 \pm 0.011$ & $0.361 \pm 0.012$ \\
Computed $(3) /(2) \ldots \ldots \ldots \ldots \ldots$ & $0.520 \pm 0.025$ & $0.564 \pm 0.022$ & $0.614 \pm 0.029$ \\
\hline
\end{tabular}

band, the correct ratio is less than $(a)$; but if the extra light is of shorter wave length, the correct ratio is greater than $(c)$. Anything but a monochromatic source in the region considered will give a higher ratio for each added filter. Still more pieces of the same filter, or, better yet, another filter with a transmission gradient of opposite sign, would settle the matter; but these were not available.

The average transmission-curves given by the maker cannot be relied upon for different melts of the same glass; hence we undertook the calibration of the UG6 filters. Our pieces were all cut from the same 2 -inch square. A most convenient monochromator was found to be the 150-foot tower telescope, with the normal solar spectrum from a 75-foot grating. A visual setting at $5168 \mathrm{~A}$ in the second order gave 10,336 $\mathrm{A}$ in the first order; with a dispersion of $0.71 \mathrm{~A} / \mathrm{mm}$, other settings were made by moving the photometer along the spectrum. The mean results for each combination of filters for three regions at intervals of $10 \mathrm{~cm}$ are in Table 2 . The differences between $(2) /(1)$ and $(3) /(2)$ are now within the accidental errors, showing that there was no scattered light and that the measured spectral regions were effectively pure. 
To utilize all the data, including some additional measures at wave lengths other than those of Table 2, two linear equations were derived expressing the ratios as functions of the wave length. The ratios from the sky, $(2) /(1)=0.565$ and $(3) /(1)=0.348$, were then substituted in these equations to get the respective wave lengths. The details are not important because of the systematic difference between the two results. We find:

$$
\begin{aligned}
\text { From }(2) /(1) \ldots \ldots \ldots & 10,427 \mathrm{~A} \\
\text { From }(3) /(1) \ldots \ldots \ldots & 10,452 \\
\text { Mean.......... } & 10,440 \pm 25 \mathrm{~A}
\end{aligned}
$$

We see no better procedure than to take the mean of the two values and to estimate the probable error to be of the same order of magnitude as the difference between them. Although the resulting wave length could be improved by further measures with other filters, it should, even under present conditions, compare favorably with anything that could possibly be obtained so far out in the infrared with a spectrograph and the present photographic plates.

\section{SPECTROSCOPIC COMMENTS}

IDENTIFICATION

The only possibilities that suggest themselves for identification of a night-sky emission near $\lambda 10440$ are: $(a)$ the forbidden ${ }^{2} \mathrm{D}-{ }^{2} \mathrm{p}$ multiplet of $N \mathrm{I} ;(b)$ the $(0,0)$ band of the $\mathrm{B}^{3} \Pi \rightarrow \mathrm{A}^{3} \Sigma$ (first positive) group of $N_{2}$.

\section{FORBIDDEN LINES OF $N$ I}

Most observers agree that the doublet near $\lambda 5200$ is absent from the night sky, as should be expected from the transition probabilities in Table 3 . They also agree that

TABLE 3

LINES OF [ $N$ I]

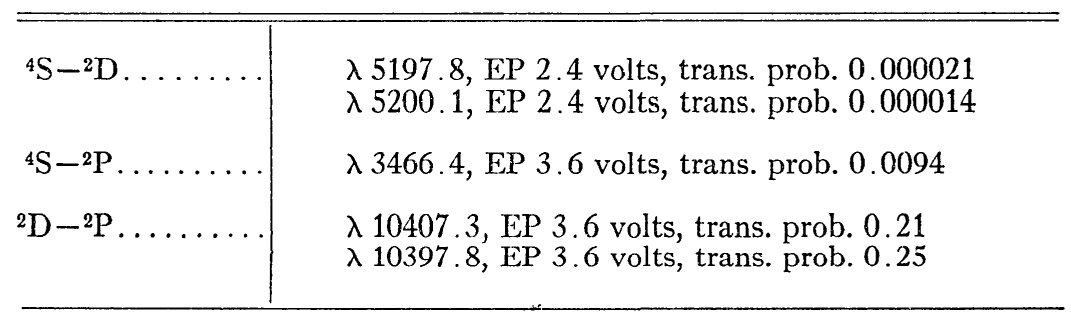

$\lambda 3466$ is likewise absent from, or hardly visible in, the night sky, although it appears in the spectrum of diffuse aurorae. The strongest [ $N_{\mathrm{I}}$ ] lines should be $\lambda \lambda$ 10407.3-10397.8, with mean wave length $\lambda$ 10402.7. The emission at $\lambda 10403$ has the same upper level as $\lambda$ 3466; hence the intensity ratio of $\lambda 10403$ to $\lambda 3466$ should be that of the transition probabilities $(0.21+0.25): 0.0094$ or $49: 1$. Although $\lambda 10403$ should thus be the strongest $[N \mathrm{I}]$ emission, it would not be able to give rise to the intense night-sky emission observed, since $\lambda 3466$ is either absent or hardly visible. It should be noted that a line fifty times the intensity of $\lambda 3466$ would still be weak, compared to the green or red $\left[O_{\mathrm{r}}\right]$ lines; and the green line is not detected by the photoelectric measurements. Hence it is safe to conclude that a $[N \mathrm{I}]$ emission cannot be a major contributor to the night-sky emission near $\lambda 10440$. Moreover, the wave length observed for the maximum intensity of the night-sky emission is found to be definitely longer than the $[N \mathrm{I}]$ line, and the difference in wave length appears greater than would be due to the experimental error. 
On the other hand, since $\lambda 3466$ is observed in certain types of aurora, a reinforcement of infrared night-sky emission in an aurora may possibly be due, at least.partly, to the contribution of $\lambda 10403[\mathrm{~N} \mathrm{r}$ ]. In such a case a shift of the intensity maximum toward the shorter wave lengths should be observed.

\section{$(0,0)$ BAND OF THE FIRST POSITIVE GROUP OF NITROGEN}

a) Wave length.-The $(0,0)$ band has been examined by A. H. Poetker ${ }^{5}$ and by A. A. Frost and $\mathrm{O}$. Oldenberg. ${ }^{6} \mathrm{~A}$ thermopile with a prism spectrometer gave a maximum at $1.042 \mu$; with a grating, four peaks were recorded between $1.035 \mu$ and $1.046 \mu$, the two strongest being at $1.041 \mu$ and $1.043 \mu$. Individual lines were observed photographically but could not be measured accurately.

Frost and Oldenberg include a reproduction of part of the $(0,0)$ band taken with a 21-foot grating in the first order, on which many individual lines are resolved; but they give no analysis of the band.

From the well-measured $N_{2}$ bands of the visual region, ${ }^{7}$ we may attempt to compute the intensity maximum of the $(0,0)$ band. But this is difficult and uncertain, especially since we ignore the temperature in the atmospheric regions where the infrared night-sky emission occurs. An estimated value of the wave length of the maximum is $\lambda 10450$. At any rate, the $N_{2}$ emission should be of wave length longer than the [ $\left.N_{\mathrm{I}}\right]$ line. The agreement in wave length between the observed night-sky emission and the $(0,0)$ band of $N_{2}$ is quite satisfactory.

b) Intensity.-The $(0,0)$ band is by far the strongest transition in the $\Delta v=0$ sequence and certainly one of the strongest bands of the whole first positive group. ${ }^{8}$

Yet, if we attribute the intense night-sky emission to the $(0,0)$ band, other transitions should be observable with fair intensity if the relative intensities were at all similar to those found in such laboratory sources as a discharge in gas. Several emissions observed in the night-sky spectrum in the region from $\lambda 5000$ to $\lambda 6000$ have been tentatively attributed to bands of the first positive group. But all these emissions are extremely weak, and many of the identifications are even very doubtful.

No appreciable emission is observed, photoelectrically, in the night sky at $\lambda 8910$, which is the maximum of the $(1,0)$ transition; this fact shows that very few $\mathrm{N}_{2}$ molecules are actually brought to the level $v^{\prime}=1$ of $B^{3} \Pi$.

Hence the identification of the night-sky emission with the $N_{2}$ band requires a mechanism enhancing the $(0,0)$ band relative to the other vibrational transitions.

c) Suggested excitation mechanism.-The value generally accepted for the heat of dissociation of $N_{2}, D\left(N_{2}\right)=7.383 \mathrm{v} .,{ }^{9}$ is based on a discussion of predissociation phenomena. Recently A. G. Gaydon ${ }^{10}$ has advocated a higher value $D\left(N_{2}\right)=9.764 \mathrm{v}$. In this discussion we shall adopt the value $D\left(N_{2}\right)=7.383 \mathrm{v}$. given by Herzberg. Actually, the excitation mechanism suggested for the $(0,0)$ band depends entirely on the correctness of this value.

The value $7.38 \mathrm{v}$. agrees almost exactly with that of the $v^{\prime}=0$ level of the $\mathrm{B}^{3} \Pi_{\mathrm{g}}$ electronic state (which is the upper state of the first positive group, $B^{3} \Pi_{g} \rightarrow A^{3} \Sigma_{u}^{+}$). In fact, the heat of dissociation of $N_{2}$ is just a little higher than $\mathrm{B}^{3} \Pi, v^{\prime}=0$, but lower than $\mathrm{B}^{3} \Pi, v^{\prime}=1$ (Fig. 1).

${ }^{5}$ Phys. Rev., 30, 812, 1928.

${ }^{6}$ Phys. Rev., 48, 66, 1935.

${ }^{7}$ For a list of these bands see, for example, Pearse and Gaydon, The Identification of Molecular Spectra (London, 1941), p. 137.

${ }^{8}$ The absence of the $N_{2}$ band in absorption in the solar spectrum is due to the high energy of $6.1 \mathrm{v}$. of the lower level $A^{3} \Sigma$.

${ }^{\circ}$ G. Herzberg and H. Sponer, Zs.f. Phys. Chem., B, 26, 1, 1934.

${ }^{10}$ Nature, 153, 407, 1944. 
Let us suppose that, during the day, $N_{2}$ molecules or other compounds containing $N$ atoms are dissociated into $N$ atoms by absorption of far-ultraviolet solar radiation. These $N$ atoms will tend to recombine in the course of the night. This will happen mainly in three-body collisions, so that the conservation of energy and momentum may be fulfilled. Since $N_{2}$ molecules constitute a major constituent of the atmosphere at any altitude, the participating third body will, in general, be an $N_{2}$ molecule. When the energy of dissociation of $7.38 \mathrm{v}$. liberated in the recombination $N+N$ is transferred to

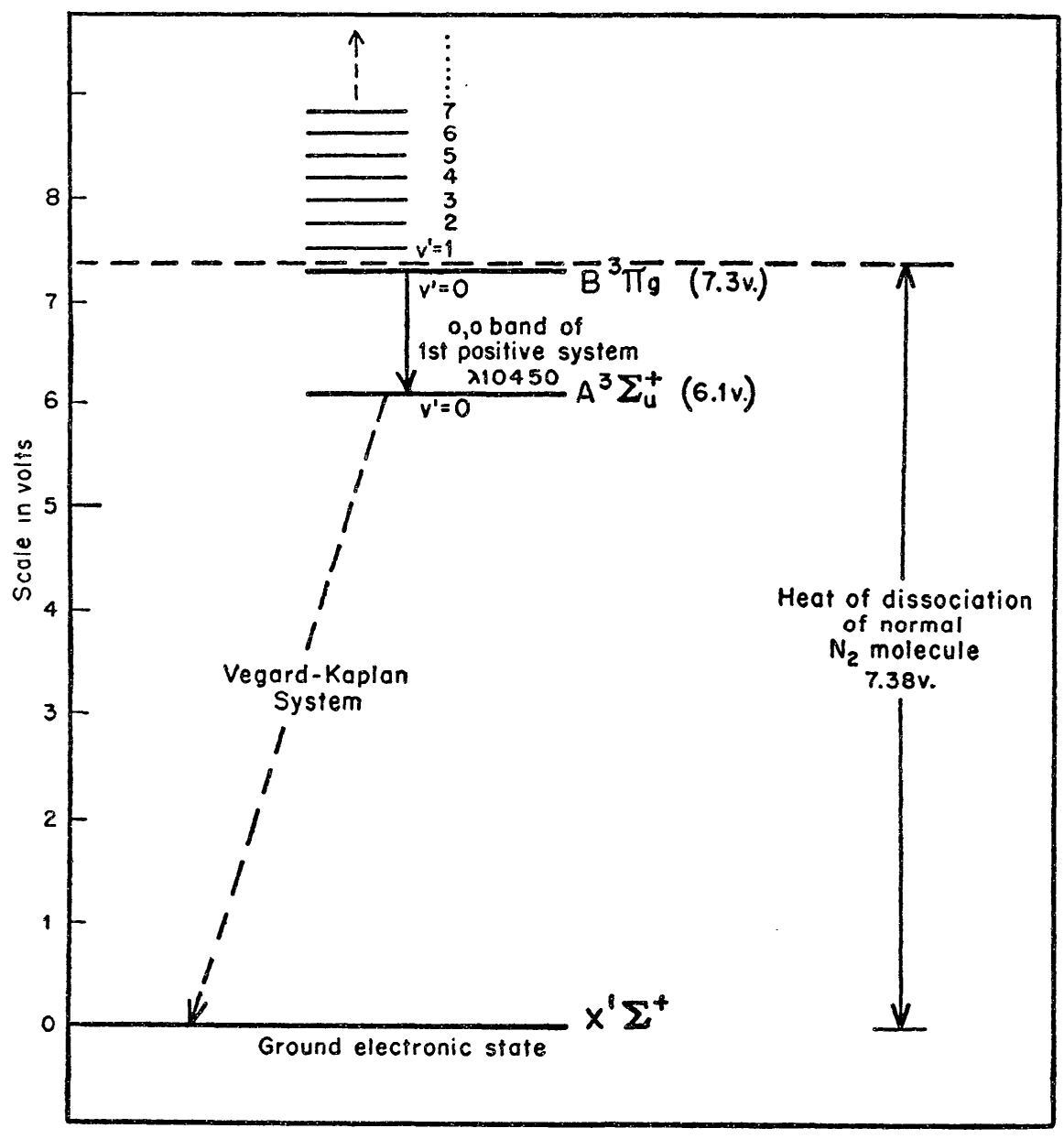

FIG. 1.-Energy-level diagram of $N_{2}$

the third body $\left(N_{2}\right)$, it will be able to excite the latter into the level $v^{\prime}=0$ of $\mathrm{B}^{3} \Pi$. Only if some additional kinetic energy is available will $v^{\prime}=1$ or higher vibrational levels of $\mathrm{B}^{3} \Pi$ become populated by this process; but the high velocity required for the $N$ atoms or $N_{2}$ molecules will be extremely infrequent even in the relatively warm regions of the upper atmosphere. Hence we should expect only the bands arising from $v^{\prime}=0$ to be intense if the suggested mechanism operates. Next in intensity to the $(0,0)$ transition is $(0,1)$ at $12,253 \mathrm{~A}$, but this would not appear in the photoelectric measurements. The third band $(0,2)$ at $\lambda 14790$ would not appear either.

The suggested mechanism of recombination at night of the $N$ atoms liberated by molecular photodissociation during the day is very similar to the mechanism suggested 
by S. Chapman ${ }^{11}$ for the $\left[O_{\mathrm{r}}\right] \cdot$ lines. Chapman suggests that the $\left[\mathrm{O}_{\mathrm{I}}\right]$ emission in the night sky is due, at least partly, to the recombination of $O$ atoms formed by dissociation of $\mathrm{O}_{2}$ during the day. Solar energy would be stored up during the day in the form of free atoms and released in the course of the night. Numerous other suggestions have been made to explain the $\left[O_{\mathrm{I}}\right]$ and other night-sky emissions. Among the latest is the mutual neutralization of $\mathrm{O}^{+}$and $\mathrm{O}^{-}$ions on impact, ${ }^{12}$

$$
\mathrm{O}^{+}+\mathrm{O}^{-} \rightarrow \mathrm{O}^{\mathrm{exc}}+\mathrm{O}^{\mathrm{exc}}
$$

an electron leaking across from the negative ion and occupying a vacant level in the positive ion.

As far as the "nonpolar" part of the night-sky emission is concerned, the observations are well explained by Chapman's mechanism..$^{13}$ The mechanism suggested here is some analogue for nitrogen of Chapman's hypothesis concerning oxygen. Evidently, a general decrease in the intensity of the infrared emission in the course of the night is readily understood in the recombination hypothesis.

It is at present impossible to predict the range in wave length covered by a $N_{2}$ band emitted in the upper atmosphere according to the mechanism suggested here, but evidently the emitted radiation is not monochromatic. It is therefore not surprising that the observed ratios $(a)$ and $(c)$ mentioned on page 3 are slightly different.

d) Objections to the mechanism of nitrogen recombination. - The photodissociation ${ }^{14}$ of $\mathrm{N}_{2}$ differs from that of $\mathrm{O}_{2}$. In fact, the difficulty of photodissociating $\mathrm{N}_{2}$ molecules is the main reason why it has usually been assumed that atomic nitrogen, if present at all in the upper atmosphere, must be there only in very small amounts. On the contrary, at great heights in the atmosphere (above $100 \mathrm{~km}$ ) most of the oxygen is supposed to be in the atomic form.

The question of the photodissociation of $N_{2}$ has recently been discussed by Ta-You Wu, ${ }^{12}$ who concludes that photodissociation of $N_{2}$ should be appreciable in the region $\lambda<500 \mathrm{~A}$, not yet explored in the laboratory. Modern evidence ${ }^{15}$ points to the fact that the sun radiates much more energy in the far ultraviolet than would a black body at $6000^{\circ} \mathrm{K}$, so that radiation would still be plentiful beyond $\lambda 500 \mathrm{~A}$. But, according to Dr. G. Herzberg, ${ }^{16}$ there is actually no need for radiation of wave length shorter than $\lambda 500$ to produce a dissociation of nitrogen. It is very likely that $N_{2}$ molecules can be predissociated by radiation of wave length shorter than approximately $1400 \mathrm{~A}$. Dr. Herzberg states that the higher vibrational levels of the $\mathrm{A}^{3} \Sigma_{\mathrm{u}}^{+}$state can almost certainly predissociate into two normal $N$ atoms. Although the transition probability from the ground state to the $\mathrm{A}^{3} \Sigma_{\mathrm{u}}^{+}$state is small, it would suffice to produce a considerable dissociation of $N_{2}$ molecules in the upper atmosphere.

Another objection to the presence of atomic nitrogen in the atmosphere is the absence of strong $\left[N_{\mathrm{I}}\right]$ emission in the night sky. Of course, to obtain a $\left[\mathrm{NI}_{\mathrm{I}}\right]$ emission,

${ }^{11}$ Phil. Mag., 23, 657, 1937.

${ }^{12}$ H. S. W. Massey and D. R. Bates, Reports on Progress in Physics, 9, 72, 1943. Incidentally, the mechanism suggested for $[O \mathrm{I}]$ by Massey and Bates would not be applicable to a $[N \mathrm{I}]$ emission, on account of the small electron affinity of the $N$ atoms. For other suggestions see Ta-You Wu, Phys. Rev., $66,65,1944$.

${ }^{13}$ C. T. Elvey, Rev. Mod. Phys., 14, 141, 1942.

${ }^{14}$ Evidently, $N$ atoms may be formed by photodissociation of molecules other than $N_{2}$, such as $N O$. But such a process is not likely to give rise to a high abundance of $N$ atoms.

${ }^{15}$ A. Hunter, Reports on Progress in Physics, 9, 5, 1943.

${ }^{16}$ Private communication. The authors wish to thank Dr. Herzberg for his helpful comments. 
appropriate excitation mechanism of sufficient efficiency must exist for the $N$ atoms. If our suggested mechanism

$$
N+N+N_{2} \rightarrow N_{2}+N_{2}^{\text {exc }}
$$

is correct, it would deplete considerably the less efficient recombination process

$$
N+N+N \rightarrow N_{2}+N^{\text {exc }}
$$

and would be much more frequent than any other excitation mechanism for $N$ I.

Wu's discussion shows that, by supposing an appreciable abundance of free $N$ atoms in the upper atmosphere, several important observations can be explained. It is possible, in this way, to reconcile the theoretical and observed densities in the $\mathrm{E}$ and $\mathrm{F}$ layers; to understand the observed recombination coefficients of electrons in these same $\mathrm{E}$ and $\mathrm{F}$ layers; and to explain the presence of $N_{2}^{+}$and $N_{2}$ bands in high aurorae. We should add that numerous reactions between $N$ atoms and oxygen or oxides of nitrogen may be of interest for the physics of the upper atmosphere.

e) Remarks.-

1. After emission of the first positive group, the $N_{2}$ molecules are left on the metastable level $\mathrm{A}^{3} \Sigma^{+}$, from which part of them will fall down toward the ground electronic level $\mathrm{x}^{1} \Sigma^{+}$with emission of the forbidden Vegard-Kaplan bands.

2. The $N_{2}$ molecules on the metastable level $\mathrm{A}^{3} \Sigma^{+}$have been invoked on numerous occasions to explain the excitation of the $\left[O_{\mathrm{I}}\right]$ lines, the peculiar behavior of the red lines of $\left[O_{\mathrm{I}}\right]$ at twilight and in certain types of aurora, etc. Many of these attempts have lost their significance following investigation and discussion by Chapman, Götz, Nicolet, and others. But some of them may still be reconsidered in the light of the present result, since the population on the $A^{3} \Sigma, v=0$ level must be appreciable.

3 . Since the mechanism of emission suggested here seems to be the only one sufficiently plausible to explain the intense night-sky emission near $\lambda 10440$, it may be considered as an argument in favor of the value $D\left(N_{2}\right)=7.38$ volts.

We are indebted to Mr. John Whittlesey for assistance in the observations for this paper. The investigation has been supported in part by grants from the Alumni Research Fund of the University of Wisconsin and from the Observatory Council of the California Institute of Technology. 